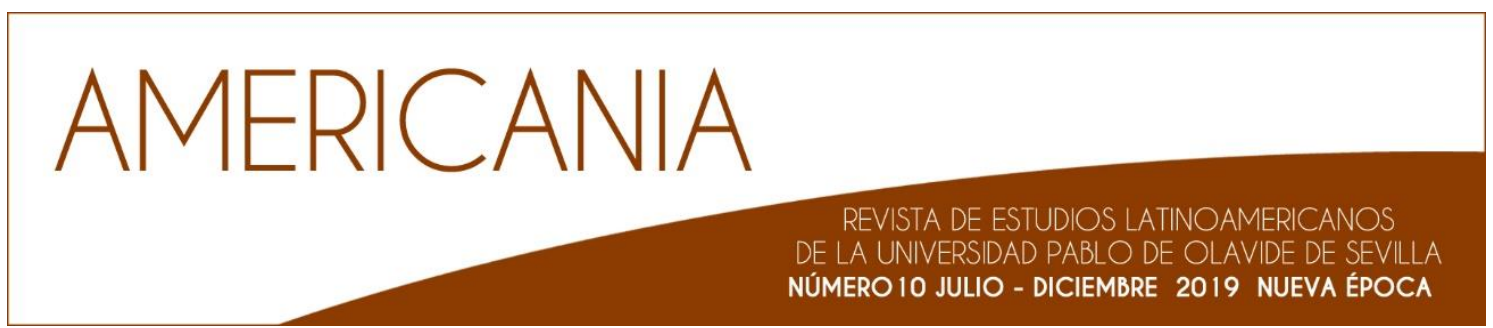

\title{
SENDERO ROJO O EL PARTIDO COMUNISTA DEL PERÚ MARXISTA-LENINISTA-MAOÍSTA (1992-1999) Ideología, Organización y Estrategia
}

\section{Resumen}

La captura en 1992 de Abimael Guzmán, líder del PCP-Sendero Luminoso, provocó un cambio significativo tanto en el conflicto armado peruano (1980-2000) como en la organización maoísta. Uno de estos cambios fue el surgimiento de un nuevo sujeto político, Sendero Rojo, también denominado PCP-Proseguir o PCPM-L-M. La existencia, primero como escisión, y segundo como partido, de Sendero Rojo comienza en 1992, se consolida en 1994-95 y se mantiene hasta 1999, con la captura de su líder, Óscar Ramírez Durand (Feliciano). El objetivo general de la presente investigación es analizar los cambios y continuidades producidos en Sendero Rojo en su surgimiento y desarrollo, así como exponer su autonomía y especificidad respecto de Sendero Luminoso. Ubicamos tres ejes centrales de la investigación: el análisis de la ideología, organización y estrategia de este grupo.

\section{Palabras Clave}

Sendero Luminoso - Guerrilla - Maoísmo - Perú - terrorismo - foquismo

Historiador y magíster en Estudios Latinoamericanos por la Universidad de Barcelona. En la actualidad, doctorando sobre el Partido Comunista del Perú, Sendero Luminoso, en la misma institución dentro del programa doctoral de Sociedad y cultura: Historia, Antropología, Artes, Patrimonio y Gestión cultural. 


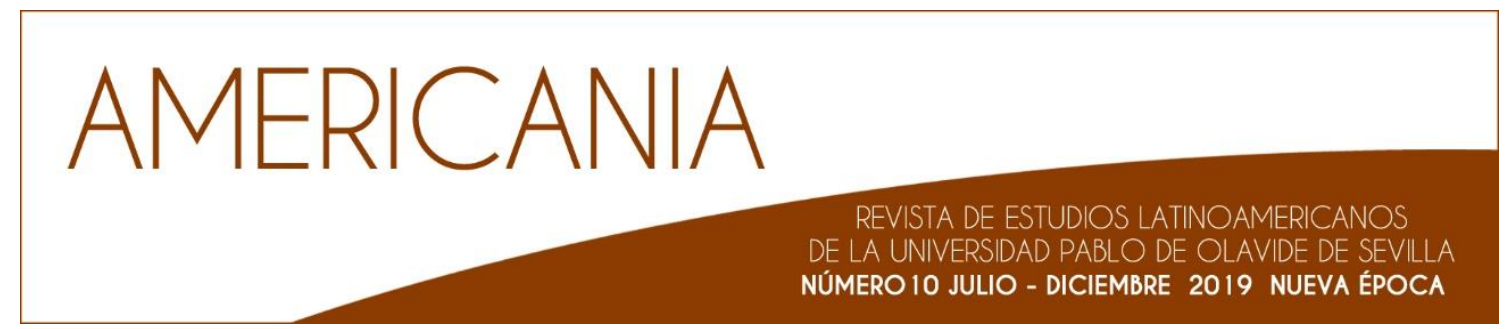

\title{
THE RED PATH OR THE PERUVIAN COMMUNIST PARTY MARXIST-LENINIST-MAOIST (1992-1999) Ideology, Organization and Strategy
}

\begin{abstract}
The capture of Abimael Guzmán, the leader of the PCP-Shining Path in 1992, provoked a significant change both in the Peruvian armed conflict (1980-200) and in the Maoist organization. One of these changes was the rising of a new political subject, the Red Path, also known as PCP-Proseguir or PCP-Marxista-LeninistaMaoísta. First as a cleavage and then as a party, the existence of the Red Path started in 1992. It was consolidated in 1995 and maintained until 1999 altogether with the capture of its leader Óscar Ramírez Durand (a) Feliciano. Consequently, the main aim of the investigation is to analyze the changes and the continuities originated by the Red Path in its rising and development as well as to expose its autonomy and specificity towards the Shining Path. There are three central axis in the investigation which are: the ideology's analysis, strategy and organization.
\end{abstract}

\section{Key Words}

Shining Path - Guerrilla - Maoism - Perú - Terrorism - Focoism 


\section{Introducción}

La captura de Abimael Guzmán Reynoso (presidente Gonzalo), en 1992, tuvo graves consecuencias en el desarrollo del conflicto armado interno que afrontó el Perú entre 1980 y 2000. Especialmente, la detención tuvo gran repercusión en el sí de la organización maoísta. En 1992 y, con más intensidad, en 1993, empezó a conformarse en el seno del Partido Comunista del Perú - Sendero Luminoso (PCP-SL2) un debate alrededor de proseguir la lucha armada o acordar la paz con el gobierno de Alberto Fujimori. Ambas posturas se erigieron como una línea interna dentro del PCP-SL. Así, a raíz de la detención del líder de Sendero, se comenzaron a desarrollar dos posturas, las cuales se convirtieron, paulatinamente, en irreconciliables.

En la presente investigación centraremos nuestro estudio en los integrantes del PCP-SL que renegaron de los acuerdos de paz y abogaron por continuar la guerra popular prolongada (GPP). Estos se erigieron, primero, como la línea 'proseguir' dentro del PCP-SL, y, segundo, una vez consumada la separación, como Sendero Rojo o el Partido Comunista del Perú, Marxista-Leninista-Maoísta. Comúnmente, tanto por la historiografía como por sus adversarios, se les denominó también como 'línea escisionista', 'bloque escisionista', 'línea liquidacionista de izquierda', 'militaristas burgueses' $\mathrm{O}$, simplemente, 'felicianistas'.

El estudio de esta organización deviene principal, puesto que no ha sido estudiado en profundidad por la literatura académica existente sobre el conflicto armado interno, ni por los demás estudios centrados en el PCP-SL. Por ello, asumimos por objetivo analizar y profundizar en el estudio de Sendero Rojo. Esto se traduce, de forma concreta, en dilucidar los cambios y procesos dialécticos existentes en la ideología; es decir, establecer las novedades y discrepancias respecto al PCP-SL, así como las aportaciones propias a la teoría y praxis. Del mismo modo, tenemos como objetivo definir cuáles fueron las nuevas formas de organización después de la captura de Abimael Guzmán, además de exponer las nuevas formas de dirigir al PCPM-L-M. Por último, pretendemos comprender el accionar y estrategia de la guerrilla

\footnotetext{
2 A lo largo del artículo utilizaremos el término PCP-SL, SL, Sendero Luminoso o Sendero, para referirnos al Partido Comunista del Perú, por el Sendero Luminoso de José Carlos Mariátegui, tanto para el período de 1971-1992, como para referirnos a las posturas 'acuerdistas', surgidas después de la captura de Abimael Guzmán. Para la escisión que surge a partir de la captura de Abimael Guzmán utilizaremos PCP- Proseguir, Sendero Rojo o PCP-M-L-M. Cuando usamos 'proseguir' hacemos mención a cómo se denominó a la línea dentro del PCP-SL que pretendía continuar la lucha armada; y tanto Sendero Rojo como el PCP-M-L-M corresponden a la denominación adoptada por su líder, Óscar Ramírez Durand (Feliciano).
} 
entre 1992 y 1999, debido al posible cambio de actuación respecto de las fuerzas del orden y la población. En definitiva, aproximarnos a un objeto de estudio que, aunque se encuentra en un periodo crucial y decisivo de la historia peruana, aún no ha sido analizado como un sujeto relevante y autónomo.

Hechas estas consideraciones, y para articular la presente investigación, hemos considerado establecer la hipótesis de que, a partir de la captura de Abimael Guzmán en 1992, la línea 'proseguir', y más tarde Sendero Rojo, entró en un proceso de abandono de la GPP, con el desarrollo de posturas propias del foquismo; produciéndose así una mutación en lo referente a la ideología, organización y estrategia. Esto provocó que Sendero Rojo tuviese un desarrollo autónomo e independiente del PCP-SL con cierta rapidez.

Para la elaboración del estudio se han utilizado tres tipologías de fuentes distintas: documentales, estadísticas y periodísticas. Las fuentes documentales hacen mención a las producidas por Wikileaks, como también a aquellas que, en forma de comunicado, produjo Sendero Rojo. Estas últimas están localizables digitalmente en el Centro de Documentación de los Movimientos Armados (CEDEMA). Las fuentes estadísticas corresponden a la documentación ofrecida por la Policía Nacional del Perú (PNP) y la Comisión por la Verdad y la Reconciliación (CVR). Las fuentes periodísticas atañen, tanto a noticias, artículos o crónicas, a prensa nacional peruana como española. El uso de estas fuentes se complementa con la bibliografía especializada sobre el tema a tratar.

Por último, la estructura de este artículo, tras esta introducción, pasa por un primer apartado donde se desarrollará el estudio de la ideología de Sendero Rojo; en el segundo apartado expondremos cómo fue la nueva forma de organizarse de la guerrilla; tercero, se analizará el accionar de la guerrilla, tanto contra las fuerzas del estado peruano como con la población civil; en cuarto y último lugar, se harán las conclusiones pertinentes y se ratificará la hipótesis. 


\begin{tabular}{|c|c|}
\hline \multicolumn{2}{|c|}{ Siglas } \\
\hline PCP-SL & Partido Comunista del Perú, Sendero Luminoso \\
\hline SL & Sendero Luminoso \\
\hline PCP-P & Partido Comunista del Perú, Proseguir \\
\hline PCP-M-L-M & Partido Comunista del Perú, Marxista-Leninista-Maoísta \\
\hline LOD & Línea Oportunista de Derecha \\
\hline CVR & Comisión por la Verdad y la Reconciliación \\
\hline PNP & Policía Nacional del Perú \\
\hline EGP & Ejército Guerrillero Pe Liberación Popular \\
\hline ELP & Valle de los ríos Apurímac, Ene y Mantaro \\
\hline VRAEM & Valle del Alto Huallaga \\
\hline VAH & Cuadro №1. Siglas \\
\hline
\end{tabular}

\section{Ideología}

Para plantear una determinada línea ideológica en un partido maoísta es necesaria la existencia de una vanguardia teórica, que sea organizada. Esta vanguardia es el organismo que se plantea las preguntas, los interrogantes y soluciona los problemas teóricos del movimiento práctico. La vanguardia ejerce de guía, pero ella sola, como también los cuadros, no pueden construir y constituir un partido. El mismo Mao Zedong define la educación ideológica (ejercida por la vanguardia) como el "eslabón clave que debemos empuñar firmemente en la labor de unir a todo el partido para la gran lucha política. De no proceder así, el Partido no podrá cumplir ninguna de sus tareas políticas"3.

En Sendero Luminoso, la vanguardia teórica la constituía el Comité Central y la Jefatura, siendo estos los órganos guías de la teoría y de la práctica revolucionaria. En 1992, el 90\% de la cúpula senderista fue capturada, es decir, la vanguardia fue desmantelada, y sin Abimael Guzmán y la cúpula, no existía el gran arbitro capaz de dirimir en las grandes polémicas político-ideológicas4. Por el contrario, el aparato

3 Zedong, Mao, El libro rojo, Espuela de Plata, Sevilla, (1964), 2014, 115.

4 Manrique, Nelson, El tiempo del miedo. La violencia política en el Perú 1980-1996, Fondo Editorial del Congreso del Perú, Lima, 2002, 237. 
militar del PCP-SL seguía relativamente indemne 5 . Este hecho es el que asienta los cimientos de la mutación ideológica de Sendero Rojo, ya que se produce un desmembramiento de la vanguardia teórica, existiendo una mayor preeminencia del aparato militar del partido. Es decir, en 1992 empezará a prevalecer lo militar a lo político.

Esta preponderancia de lo militar hacia lo político tiene como eje central el desplazamiento de la dirección del Partido hacia el campo, a partir de 1992. Los nuevos dirigentes, especialmente, Feliciano, vivían, participaban y lideraban directamente la lucha armada en las zonas rurales. Esto hizo que lo militar adquiriese preponderancia. De hecho, con anterioridad a la captura de Guzmán ya existió en el seno del PCP-SL una lucha interna entre la vertiente más militarista, que tenía como tesis el aumento de las acciones en el campo (liderada por Feliciano y Morote6) contra la línea que pretendió mantener e incrementar la importancia de la ciudad, seguida por Guzmán y la mayoría del Partido. Un ejemplo del debate lo hallamos en la acusación de 'hechismo7' que hizo Guzmán a Feliciano, debido a que este último rechazaba la separación de dirección de los hechos guerrilleros, es decir, de la primera línea de combate. La constatación de tales planteamientos vendrá dada por el incremento de las acciones del terrorismo revolucionario, como el asesinato selectivo ${ }^{8}$, y de la retórica aún más belicista y militarista ubicada en los textos del PCPP.

Por este motivo, la elaboración de textos senderistas después de la captura de Guzmán fue en descenso. Este es un fenómeno que se produce a largo plazo ya que, en los años inmediatos, de 1992 a 1996, tuvieron lugar una gran cantidad de comunicados?. Esto es debido al intento de recomposición llevado a cabo por el Comité Central de Emergencia en favor de la guerra popular prolongada, de proseguir en la lucha pese a los acuerdos de paz. Se ha dado constancia del intento

5 Ibid.

6 Osmán Morote Barrionuevo fue un importante cuadro de Sendero Luminoso. Su adhesión al PCP-SL data desde los inicios del partido, siendo uno de los fundadores. Se le creía el número dos de la organización. Fue capturado en 1989.

7 Jiménez, Benedicto, Inicio, desarrollo y ocaso del terrorismo en el Perú, 2 vols., Sanki, Lima, 2000, 2:186.

8 En el III Pleno del PCP-SL se adoptó un reajuste de las formas lucha, y esto significó eliminar el llamado 'aniquilamiento selectivo' y ceñirse al artículo tercero común de la Convención de Ginebra. Sendero Rojo, en desacuerdo con lo anterior, siguió un camino paralelo al continuar tales prácticas en sus actividades armadas.

9 Los más destacados son: iSuperar el recodo, desarrollando la guerra popular!; Informe político: Unir al pueblo contra la dictadura fascistas, genocida y vendepatria, desarrollando más la guerra popular; iViva el Partido Comunista de Perú!; y, finalmente, iPresos políticos libertad! 
de reorganización de la vanguardia teórica por parte de las filas senderistas. En concreto, en el año 1992, en la provincia de Ayacucho, se produjo una reunión de los principales mandos senderistas, así como también en la ciudad de Lima, donde en 1995 se realizó otra reunión de importantes cuadros 10. De esta reorganización a nivel nacional surgirán los dirigentes del PCP-Proseguir.

Del mismo modo, entre los años 1992 y 1996 hallamos numerosas detenciones sobre cuadros que participaban en la reorganización de la vanguardia teórica del partido, por lo que nos lleva a sostener que estos arrestos denotan la intencionalidad de Sendero Rojo por reafirmarse como partido en lo ideológico. Las detenciones, sobre todo, se produjeron en el Comité Metropolitano de Lima y de Socorro Rojo ${ }^{1}$. La lucha ideológica y el intento de 'proseguir' por expandir su nueva doctrina no solo pasó por la producción panfletaria y propagandística, sino que se convirtió en la confrontación directa mediante el uso de la violencia. El suceso paradigmático de la lucha por la independencia ideológica y, sobre todo, por la lucha por conseguir la influencia de los senderistas dubitativos, fue el asesinato y dinamitación del senderista pro-Guzmán 'Negro José'12.

Es oportuno señalar que los comunicados estudiados están firmados por el Comité Central del PCP y, a partir de 1996, aparecen firmados por el PCP-Base Huallaga, dato que corrobora que, al menos entre 1992 y 1996, existió cierta organización centralizada de donde saldrían las principales directrices ideológicas de Sendero Rojo.

Asimismo, podemos relacionar el auge de los comunicados con los periodos de mayor intensificación de la guerrilla y el aislamiento e independencia se consuma cuanto más es arrinconado el PCP-M-L-M hacia el Huallaga. Esta reorganización inicial se traduce en un proceso de mutación ideológica respecto a las tesis del PCP-SL, puesto que entran en escena nuevos debates, estrategias, tácticas y análisis sobre la situación nacional del Perú. Por tanto, aseguramos que después de la captura de Guzmán los escisionistas mantuvieron la máxima comunista de que "no puede haber teoría revolucionaria sin movimiento revolucionario"13, dado que varió su análisis

10 Torres, León-Jimmy, Caretas, Lima, 8 ago. 1996; Montoya, David, Caretas, Lima, 15 jul. 1999.

11 Caretas, Lima, 15 ago. 1996; Caretas, Lima, 10 sep. 1998; Caretas, Lima, 9 may. 1996; Rospigliosi, Fernando, Caretas, Lima, 23 may.1996.

12 Lama, Abraham, Inter Press Service, Lima, 16 may. 1996.

13 Lenin, Vladimir Ilich, ¿QueHacer? Debarris, Barcelona, (1902), 2013, 44. 
materialista sobre la guerra popular y la situación del Perú. Otra cuestión bien distinta reside en si estas nuevas aportaciones se alejan del marxismo-leninismo-maoísmo pensamiento Gonzalo, como veremos a continuación.

Pese a la comentada reorganización de SL a partir de 1992, no hallamos una innovación teórica hasta 1995, con el cambio del Plan Estratégico Militar. De 1992 a septiembre de 1995, la línea 'proseguir' 14 y posteriormente Sendero Rojo mantuvieron como eje central de su ideología y accionar los principios esgrimidos por Guzmán en el llamado 'Equilibrio Estratégico'15. En consecuencia, este lapso constituye una transición entre lo nuevo y lo viejo.

La continuidad del mismo plan de acción después de la caída de la cúpula también significó una continuidad de las acciones terroristas revolucionarias en los años inmediatos a la captura, entre 1992 y 1995. Aunque existió un leve descenso de las acciones, se mantuvo cierta capacidad de acción muy cercana a la de los mayores años de violencial6 (1989-1992). Por tanto, se produjo una correlación entre la invariabilidad de la ideología y el mantenimiento de la actividad armada; por el contrario, cuando la actividad armada fue en descenso, a partir de 1995 y, sobre todo, en 1996, la separación ideológica se hizo mayor. Esta tesis viene reforzada por el trabajo político e ideológico que se vivió en prisión, puesto que existió una diferenciación consumada entre partidarios de Feliciano y Guzmán ya en 199517. Así, a medida que la organización sufrió reveses, como comentaremos en el siguiente apartado, cambió su línea política y militar.

La ruptura con el PCP-SL es expuesta con claridad en el comunicado "Superar el recodo, desarrollando la guerra popular". Este texto ratifica que el PCP-M-L-M se refuerza ideológicamente y abre una nueva etapa, planteamiento este similar al sostenido, aun siendo sucintas sus aportaciones, por autores como Burt y Riccil8 y

14 La Facción y línea 'proseguir' corresponde al período de 1992-1995, antes de que se separase definitivamente del PCP-SL, ya que no constituía un partido separado, sino más bien una tendencia propia dentro de Sendero Luminoso. Así que a partir de 1995 podemos hablar de PCP-Proseguir, PCP-M-L-M o Sendero Rojo.

15 El ‘Equilibrio Estratégico' fue declarado después del Primer Congreso del PCP-SL, entre 1988 y 1989. Esto significaba que las fuerzas de Sendero habían llegado a equipararse con las del aparato estatal, lo que se tradujo en un aumento considerable de las acciones guerrilleras, provocando una mayor exposición de los senderistas hacia las fuerzas del orden. El 'Equilibrio Estratégico' también ha sido entendido como uno de los causantes de la derrota de Sendero.

16 Policía Nacional del Perú (PNP), "Perú: estadísticas de violencia y seguridad 1998 - 1999", PNP, 1999, http://proyectos.inei.gob.pe/web/biblioineipub/bancopub/Est/Lib0061/indice.htm (Consultado el 10/01/2018).

17 Rénique, José Luis, La voluntad encarcelada. Las 'luminosas trincheras de combate' de Sendero Luminoso del Perú, Instituto de Estudios Peruanos, Lima, 2003, 110-113.

18 Burt, Jo-Marie y López Ricci, José, “Perú: Shining Path after Guzmán”, NACLA Report on the Americas, v.3, n.28, 1994 6-9. 
Antezana'19. La ruptura la encontramos en la evolución consciente del 'Equilibrio Estratégico' y de la captura de la vanguardia teórica. Este nuevo plan consta de 6 objetivos básicos, que sirven de síntesis de cómo pretendía el PCP-M-L-M diferenciarse del pasado y, sobre todo, legitimarse. En concreto, Sendero Rojo sostenía los siguiente:

"1. Superar el recodo de la captura de Guzmán. 2. Combatir la reacción y el Imperialismo. 3. Aplastar la patraña de la "pacificación" 4. Aplastar la LOD20, revisionista y capitulacionista, 5. Sentar las bases para la realización del IV Pleno. 6. Reorganización general del partido"21.

De forma más concreta, en el texto "Informe Político" se abre veda de los errores del pasado del PCP-SL, específicamente del equilibrio estratégico, entrando así abiertamente en seria diferencia con el legado senderista. Incluso, se llegará a afirmar que:

"Es necesario superar errores y limitaciones como la subestimación o sobreestimación del enemigo, ver objetivos y aplicarlos como corresponde el Gran concepto Estratégico, no menospreciar tácticamente al enemigo, ni siquiera a las mesnadas [...] Los errores cometidos hacen daño y pueden ser usados por la reacción. De allí la exigencia de no afectar en ningún momento los intereses del pueblo y que toda acción tenga claro y definido sentido político"22.

Como se ha observado, el PCP-Proseguir erige claramente un distanciamiento y autocritica respecto de su predecesor, el PCP-SL, para legitimar y proseguir sus acciones, debido a que han corregido la línea 'errónea' de abandonar la lucha armada.

En añadido, este planteamiento entra en contradicción con el comunicado "Instrucciones" y "iContra la dictadura genocida y vendepatria, persistir en la guerra popular!". En ambos textos se expone, explícitamente, que debe aumentar la belicosidad de las acciones y del discurso, hecho que contrasta con las palabras

19 Antezana, Jaime, “El senderismo real está en el Movadef", Perú21, 2009, https://peru21.pe/politica/jaime-antezanasenderismo-real-movadef-193743 (Consultado el 03/01/2018).

20 Línea Oportunista de Derecha. Aquí el texto hace mención de los senderistas que están en favor del Acuerdo de Paz con el gobierno fujimorista.

21 PCP-M-L-M, "iSuperar el recodo, desarrollando la guerra popular!", PCP-M-L-M, 1995, http://www.cedema.org/ver.php?id=3527 (Consultado el 29/03/2018).

22 PCP-M-L-M, “Informe político", PCP-M-L-M, 1996, http://www.cedema.org/ver.php?id=723 (Consultado el 29/03/2018). 
citadas anteriormente de no precipitar las acciones. La realidad demostró que no hubo un cambio consustancial entre sus proclamas y la violencia ejercida ${ }^{23}$. En este sentido, debemos recordar el rechazo del PCP-SL hacia atentados en los que se exponían víctimas civiles, especialmente después del denominado 'error' de Tarata 24. La importante relevancia de dicho atentado propició un cambio de línea, al menos en la retórica, sobre la modalidad del atentado, por las nefastas consecuencias sobre las masas populares. En contraposición, el comité central de Sendero Rojo incide en "avanzamos en elevar la belicosidad. Tenemos tres tareas: combatir, movilizar, producir. Menos, pero mejores tropas y una administración más simple"25.

En esta afirmación se mantiene la contradicción entre belicosidad y rectificación del pasado, puesto que no existió un cambio significativo entre uno $u$ otro partido en los primeros años 26 . Específicamente, el aumento de la belicosidad, expresado de forma sistemática por Feliciano, tuvo sus inicios desde bien temprano, en 199327. De la afirmación también encontramos un elemento esencial: el militarismo. La línea política y militar propuesta por el PCP-M-L-M está centrada en reactivar las acciones militares y continuar con la guerra popular. Si hemos comentado que hubo una continuación de las acciones y no se innovó en el discurso, podemos sostener que el fusil mandó sobre el partido y que, hasta 1995, Sendero Rojo, en contradicción con Mao, "no inició una investigación y un análisis básicos del número de los partidarios activos, de los opositores y de los que mantienen una posición intermedia, y no tomar decisiones sin fundamento ni de manera subjetiva"28.

La tesis del militarismo se halla claramente en el comunicado "Instrucciones", donde se cita de forma reiterada el planteamiento de Lenin en la Guerra Civil Rusa sobre la necesidad de armar al proletariado y de "tener en cuenta que ahora, en la preparación y en la instrucción, revisten mucha más importancia las propias acciones

23 CVR, Informe Final, Comisión de la Verdad y Reconciliación, Lima, 2003.

24 El atentado de Tarata ocurrió el 16 de julio de 1992, cuando un coche-bomba, estacionado en medio de la calle Tarata, en el distrito de Miraflores, explotó. El atentado produjo 26 víctimas mortales. Sobre la autoría del atentado han existido múltiples debates. Abimael Guzmán y el Comité Central de Sendero Luminoso en 1992 criticaron en documentos partidarios la colocación del explosivo. En el juicio que acusaba a la cúpula de Sendero del atentado, estos negaron que tuviesen una relación directa con el atentado.

25 PCP-M-L-M, “Informe político", PCP-M-L-M, 1996, http://www.cedema.org/ver.php?id=723 (Consultado el 29/03/2018).

26 Policía Nacional del Perú (PNP), "Perú: estadísticas de violencia y seguridad 1998 - 1999”, PNP, 1999. http://proyectos.inei.gob.pe/web/biblioineipub/bancopub/Est/Lib0061/indice.htm (Consultado el 10/01/2018).

27 Jiménez, Benedicto, Inicio, desarrollo y ocaso del terrorismo en el Perú, 2:166.

28 Zedong, Mao, El libro rojo, 104. 
militares"29. Por lo tanto, Sendero Rojo puede considerarse como un partido-ejército. Empero, a partir de 1995 el PCP-M-L-M elabora la originalidad a raíz del nuevo plan. Pararemos atención a tres innovaciones discursivas: el Ejército de Liberación Nacional el Imperialismo y la actitud hacia el presidente Gonzalo.

La fuerza principal de Sendero Luminoso fue el EGP30. El PCP-M-L-M cambiará la denominación de este, otorgándole por nombre el de EPL ${ }^{31}$. Este cambio de nombre tiene por explicación la nueva argumentación del PCP-M-L-M sobre la situación nacional del Perú. En un primer momento, este cambio de siglas nos indica claramente, por la similitud con otros movimientos de nombre similar en Latinoamérica, que se hace frente a una intervención extranjera, concretamente de los Estados Unidos. También creemos que hace mención explícita a la guerrilla antijaponesa que se originó en China a raíz de la invasión nipona. Esto tendría por objetivo agrupar a un mayor número de personas y crear un 'Frente Único', Sendero Rojo a la cabeza, contra una agresión imperialista estadounidense, la cual produciría un mayor poder y protagonismo a la organización maoísta. En este sentido, el PCP-ML-M deseaba que Dios estuviese demasiado lejos y la intervención de Estados Unidos demasiado cerca, así lograrían su salvación y permanencia. El planteamiento de esta postura es de carácter mecanicista, antidialéctica y fatalista32, ya que asumen que el porvenir del Partido depende de un factor externo y no de la propia conciencia del partido. En 1995 definían claramente que existiría una intervención americana en el Perú:

"Siglo XX ¿̇Cómo estamos? En este siglo XX hay un imperialismo que nos domina, principalmente el norteamericano, esto es real, todos lo saben, no lo pueden callar. ¿ Y a dónde nos ha traído? ya sin recordar aquellos años 20, aquí y ahora en la peor crisis de toda la historia del estado pervano. Sacando la lección de siglos anteriores, ¿Qué cosa se puede pensar? Otra vez la nación está en riesgo, otra vez la república está en riesgo, otra vez el territorio está en riesgo puede ser perdido fácilmente y por intereses"33.

29 Lenin, Vladimir llich en PCP-M-L-M, “iQue la mitad que sostiene el cielo sea ejemplo una vez más, Hoy en la nueva etapa del partido!" PCP-M-L-M, 1994, http://www.cedema.org/ver.php?id=3527 (Consultado el 29/03/2018).

30 Ejército Guerrillero Popular

31 Ejército Popular de Liberación

32 Lenin, Vladimir Ilich, Materialismo y empiriocriticismo, Grijalbo, Barcelona, (1908), 1975, 190-191.

33 PCP-M-L-M, "iContra la dictadura genocida y vendepatria, persistir en la guerra popular!", PCP-M-L-M, 1995, http://www.cedema.org/ver.php?id=718 (Consultado 30/03/2018). 
Del mismo modo, el gobierno peruano, encabezado por Fujimori, es considerado como un títere del Imperialismo y, sobre todo, se le describe como fascista. Esta posición de continua beligerancia con Fujimori chocaría con el respeto fundado de Abimael Guzmán en la relación epistolar que mantuvo con Montesinos y con el presidente. Como venimos observando, la creación ideológica del PCP-M-L-M está fundada en contraposición con el PCP-SL.

Por último, cabe señalar que, pese a la animadversión que sentía el PCP-SL respecto de la denominada 'LOD', la figura de Guzmán aún seguía siendo respetada, ya que era el guía del pensamiento. La noción sobre el presidente Gonzalo, aun así, ya no se escuda en obedecer sus órdenes, puesto que creen que han manipulado su figura, sino que toman únicamente su pensamiento.

\section{Organización}

La organización Sendero Rojo presenta una continuación con Sendero Luminoso en aspectos tales como la dirección interna y el modo de organización a nivel nacional, caracterizada por la clandestinidad y la jerarquización. Como elementos específicos del PCP-M-L-M señalaríamos la regionalización de la dirección, la ausencia de una jefatura y la existencia de un nuevo organismo, el 'Comité Central de Emergencia'. Este nuevo órgano regidor del partido surge, como ya comentamos en el apartado de ideología, fruto de la reorganización senderista después de la caída de Abimael Guzmán en 1992. El Comité Central de Emergencia actuaba como la anterior 'Dirección Central'. En él se reunían los distintos líderes y representantes de los comités zonales y regionales de toda la geografía peruana. Dicho órgano ejercía de dirección bajo el liderazgo de Feliciano. Sin embargo, Feliciano no tenía una preeminencia totalizadora sobre los órganos similar a la de Abimael Guzmán, ya que no ejercía de 'presidente' ni tenía la suficiente capacidad para dar y crear una determinada línea política y militar, especialmente política. En consecuencia, la forma de organizar de Feliciano estuvo basada en dar 'directivas'34 militares, quedando muy lejos del modelo comunista del centralismo democrático.

Cabe mencionar que este sistema, digamos que 'centralizador y único', existió

34 Jiménez, Benedicto, Inicio, desarrollo y ocaso del terrorismo en el Perú, 2:197. 
de forma eficiente hasta 1996, fecha en la cual el partido se replegó hacia el campo. Las acciones coordinadas que demuestran cierta organización centralizada las hallamos en el conjunto de atentados en todo el país en las elecciones municipales de 199335 y generales de 199536. Estos hechos demuestran cierta coordinación antes del repliegue en el campo. La nueva organización del PCP-Proseguir tuvo como nombre 'Nueva Red', organismo que intentó recuperar las antiguas estructuras del PCP-SL, como también impulsar acciones de carácter jurídico y de apoyo internacional. Aun así, y en coincidencia con el desplazamiento al campo de los guerrilleros, fue desarticulada en 199537.

A partir de 1996, las acciones armadas se desplazaron predominantemente hacia regiones rurales de difícil acceso. Esta nueva situación influyó en la organización, así se estableció un mayor carácter autónomo e impermeable (entre senderistas) que el presentado por el PCP-SL. Es decir, los Comités regionales, órganos directores en el interior del país, experimentaron una mayor independencia. Esta independencia se produce por la regionalización del PCP-M-L-M, dado que el establecimiento en zonas selváticas y recónditas implicó una mayor dificultad comunicativa. A esto, pues, se le añadió el cerco policial y la caída progresiva de cuadros, hechos que hacen pensar en que el partido de 'los mil ojos y los mil oídos' tenía, más bien, una visión parcial. Empero, es cierto que existieron lazos organizativos constatados entre los focos guerrilleros de los departamentos de Junín, San Martín y Ayacucho, puesto que Feliciano se movía con facilidad por las nombradas zonas ${ }^{38}$.

La última reunión del PCP-M-L-M de la que hemos tenido constancia fue la producida en Ayacucho en 1996, que finalizó con la captura de Elizabeth Cárdenas Huyta (Aurora), miembro del Buró Político39. En esta información hallamos elementos esenciales: las fuerzas del orden tenían constancia de los movimientos senderistas y la retirada al campo coincide con la última reunión. Por ello sostenemos que la estrategia defensiva de los líderes senderistas rojos está intrínsecamente relacionada con la detención de sus miembros más importantes, por lo que la organización se

35 En las elecciones municipales de 1993, tanto la facción proseguir como el PCP-SL llamaron al boicot electoral y al sabotaje del proceso. Si bien hubo una coordinación a nivel nacional para provocar atentados y acciones de boicot, no supusieron un desafío real al fujimorismo. Del mismo modo sucedió en las elecciones generales de 1995.

36 Ibarz, Joaquim, La Vanguardia, Barcelona, 29 ene. 1993, p. 4; Ibarz, Joaquim, La Vanguardia, Barcelona, 6 abr. 1995, p. 8.

37 Jiménez, Benedicto, Inicio, desarrollo y ocaso del terrorismo en el Perú, 2:27-28.

38 Montoya, David, Caretas, Lima, 15 jul. 1999.

39 El País, Madrid, 8 dic, 1996. 
trasladó a las zonas alejadas de forma consciente.

La dinámica de detenciones de altos miembros senderistas fue progresiva. Así, en 1995 se capturan a dos líderes muy relevantes, Pedro Quinteros Ayllón y Jenny Rodríguez Neyra, ambos mandos políticos y militares de nivel nacional; también fue significativa la captura de Marge Clavo Peralta y de su esposo Olivares del Campo40; incluso, en el día posterior a la captura de Feliciano, en julio de 1999, fueron detenidos relevantes cuadros como Olga, Rita y Raquel41.

En suma, este seguido de detenciones nos muestran que el debilitamiento de Sendero Rojo era una realidad. Si en 1992 el PCP-SL quedó muy debilitado pese a ser un partido extremadamente organizado, las detenciones de líderes de 'proseguir', en un contexto desfavorable y de defensiva, no podían más que significar un debilitamiento consumado, teniendo en prisión, de forma progresiva, a gran parte de sus dirigentes principales. En añadido, la ausencia de un plan a largo plazo o de una ideologización como la del PCP-SL también ayudó a debilitar a la organización por la ausencia de una cohesión. En líneas generales, y en base a los datos proporcionados por el ordenador de Abimael Guzmán localizado en 1990, el PCP-SL contaba con una fuerza principal de 816 efectivos, una fuerza local de 4.674, y una fuerza base de 17.940, con un total de 23.430 miembros $^{42}$. Únicamente contaban con 6.000 o 7.000 efectivos con alguna capacidad verdaderamente apta para el combate en operaciones militares ${ }^{43}$. Es decir, las fuerzas capacitadas para entrar en combate contra el ejército eran un porcentaje muy bajo del total; además, estas se encontraban en un $80 \%$ entre Ayacucho, Junín y Pasco.

Teniendo en cuenta este panorama global antes de 1992, observamos cómo cambió y fue menguando la organización militar de 'proseguir' y Sendero Rojo. Después de los acuerdos de paz, la fragmentación entre proseguir y abandonar la lucha armada fue un hecho y esto, traducido en números, significa que Feliciano contaba con un total de 400 efectivos, y Guzmán con 10044. En consecuencia, hallamos que solo permanecieron 'fieles' y no abandonaron la causa los miembros con una mayor implicación directa con el partido, puesto que el grueso de las fuerzas

40 Montoya, David, Caretas, Lima, 15 jul. 1999.

41 Montoya, David, Caretas, Lima, 22 jul. 1999.

42 Caretas, Lima, 4 abr. 1996.

43 Ibid.

44 Ibid. 
lo componían 'bases de apoyo' y 'milicias', es decir, campesinos que se situaban en zonas con presencia senderista. Esta es, pues, una de las especificidades organizativas que atañen a los senderistas rojos, el constreñimiento de sus filas con preponderancia exclusiva de cuadros comunistas, que pueden ser identificados con el EPL casi exclusivamente.

Si comparamos estos datos con el número estimado que existía para cada Comité en 1994 (cada columna eran 100 hombres), se observa que existían 1.200 efectivos para todo el territorio nacional45. Esto nos indica la dinámica de disminución progresiva de la capacidad militar de Sendero Rojo. En concreto, Sendero Rojo tenía como una de sus principales acciones la incursión en poblados, las cuales se efectuaban en columnas de no más de 50 senderistas, sobre todo en las zonas del Huallaga, algo que confirmaría la tendencia a la disminución de efectivos. A pesar de la visible fragmentación, entre 1996 y 1998, todavía mantenían un número elevado de acciones armadas y, aproximadamente, contaba con 300 y 350 efectivos ${ }^{46}$. Así pues, el PCP-M-L-M se fragmentaría en cuanto a organización política, pero mantendría una tropa fiel y estable, sin grandes variaciones.

Hasta 1996 Sendero Rojo mantuvo una ideología y una estructura organizativa similar a la del PCP-SL. A partir de entonces, con el progresivo arrinconamiento de sus fuerzas, la organización no supuso un grave peligro para el estatus quo del Perú, fruto de su debilitamiento progresivo, pero sí tuvo incidencia su regionalización. Tal y como declaraba el rondero Edilberto Oré Cárdenas, del pueblo de Jochacc, provincia de Huanta, departamento de Ayacucho, "aunque el gobierno festeje la captura de Feliciano, nosotros no estamos tranquilos. Somos conscientes que la estabilidad del país no peligra, pero síla de estos poblados"47. La regionalización organizativa fue un hecho, como también la regionalización de los problemas derivados de los restos del conflicto interno peruano.

\section{Estrategia}

"La estrategia es el uso del encuentro para alcanzar el objetivo de la guerra.

45 Montoya, David, Caretas, Lima, 22 jul.1999.

46 Lama, Abraham, Inter Press Service, Lima, 20 jul. 1999; Lama, Abraham, Inter Press Service, 5 oct. 1999; Caretas, Lima, 23 abr.1998; Caretas, Lima, 1 oct. 1998.

47 Edilberto Oré Cárdenas en Montoya, David, Caretas, Lima, 22 jul. 1999. 
Propiamente hablando, sólo tiene que ver con el encuentro, pero su teoría debe tener en cuenta, al mismo tiempo, al agente de su propia actividad, o sea, las fuerzas armadas, consideradas en sí mismas y en sus relaciones principales; el encuentro es determinado por éstas y, a su vez, ejerce sobre ellas unos efectos inmediatos. El encuentro mismo debe ser estudiado en relación tanto con sus resultados posibles como con las fuerzas espirituales y del carácter, que son las más importantes en el uso de ese encuentro".

"La estrategia es el uso del encuentro para alcanzar el objetivo de la guerra. Por lo tanto, debe imprimir un propósito a toda la acción militar, propósito que debe concordar con el objetivo de la guerra. En otras palabras, la estrategia traza el plan de la guerra y, para el propósito aludido, añade la serie de actos que conducirán a ese propósito; es decir, traza los planes para las campañas por separado y prepara los encuentros que serán librados en cada una de ellas $48 . "$

Estas eran las primeras palabras del teórico Carl Von Clauswitz sobre la estrategia militar. Serán estas palabras las que emplearemos para asentar los pilares del presente apartado, como lo son las motivaciones del accionar militar, las formas en que se llevan a cabo los enfrentamientos, la modalidad de la acción militar y del terreno. Sobre estos cimientos descansa nuestro análisis sobre la estrategia seguida por Sendero Rojo después de la caída de Abimael Guzmán. Estos elementos nos permitirán definir dos fases estratégicas en el PCP-M-L-M: una de 1992 a 1995, y otra de 1995 a 1999. El análisis que presentamos a continuación se basa en la información proveniente de la PNP y por la CVR.

Esta primera etapa constituye, del mismo modo que sucedió con la ideología, una continuidad con el legado del PCP-SL. La 'guerra popular hasta el comunismo' y el 'Equilibrio Estratégico' seguían siendo la estrategia que seguir. Pero debemos plantearnos si la captura de Guzmán, en términos estratégicos, fue considerada como una derrota para los seguidores de 'proseguir'. Estos creyeron que la captura de Guzmán era parcial, siguiendo el esquema de Mao de que "la derrota nos es más que parcial y temporal, porque la destrucción total del Ejército Rojo no puede ser considerada como una derrota completa en la Guerra Revolucionaria, pero eso

48 Clauswitz, Carl Von, De la guerra, Labor, Barcelona, (1832), 1992, 171. 
jamás ocurrido"49. Efectivamente, caído Guzmán, aún persisten los atentados, y la maquinaria de guerra del terrorismo revolucionario sigue operando después de 1992.

Tal y como expone Mao Zedong, cuando se produce una derrota parcial, debe llevarse a cabo una retirada estratégica50. La facción 'proseguir', como acabamos de señalar, se mantuvo en activo al no considerar que estaba en una derrota total. Así pues, no consideraron la captura de Guzmán como una derrota que mereciese una retirada estratégica, de modo que aceptaron la confrontación directa contra el Estado. Esto significaba la sobreestimación de sus propias fuerzas y la subestimación de las fuerzas del enemigo, puesto que se negaban a adoptar medidas defensivas que permitiesen iniciar un repliegue o replanteamiento de la estrategia de la actividad armada. Por el contrario, plantearon una situación de ofensiva estratégica contra el Estado, sin contar con una ideología ni estrategia en el largo plazo. La situación objetiva era de retroceso, y 'proseguir' planteó que lo subjetivo era continuar.

Si analizamos las acciones subversivas totales de 1992 a 1995, observamos que existe un declive de estas a partir de 199251, pero aun así se mantiene un número significativo de acciones al situarse por encima de los mil atentados anuales, cifra nada despreciable. Encontramos un comportamiento similar con el total de víctimas y desaparecidos de 1992 a 1995 y con el cómputo general de delitos por terrorismo, puesto que se mantienen, aunque de forma inferior, datos similares a los años anteriores a 199252. Esto se acrecienta si nos fijamos detenidamente en el principal foco de las acciones senderistas: las áreas urbanas.

Desde la segunda mitad de la década de los 80, Sendero había puesto en su mira las zonas urbanas, con especial énfasis en Lima. La facción 'proseguir', en esta etapa, será una fiel seguidora de dicha estrategia. La actividad urbana hasta 1995 mantuvo una tendencia similar a la producida por el PCP-SL. Esto nos sirve, incluso, para afirmar que se mantenía una estrategia y una capacidad organizativa más elevada de la que se podía esperar después de la caída de la cúpula. Materializamos este planteamiento con la gran cantidad de víctimas y desaparecidos en áreas

49 Tsé-Tung, Mao, La guerra revolucionaria, Grijalbo, México, (1936), 1971, 55.

50 Tsé-Tung, Mao, La guerra revolucionaria, 64.

51 Policía Nacional del Perú (PNP), "Perú: estadísticas de violencia y seguridad 1998 - 1999”, PNP, 1999. http://proyectos.inei.gob.pe/web/biblioineipub/bancopub/Est/Lib0061/indice.htm(Consultado el 10/01/2018).

52 Ibid. 
urbanas de 1993 al 200053. De hecho, comparando los porcentajes, el PCP-M-L-M produjo más víctimas en el período de 1993 a 2000 que para el período de 1989 a 1992 e igual que en los años 1983 y 198554, y menos que durante el periodo de máxima violencia de los años 1986-1988.

Analizando por separado de la dinámica general del conflicto armado entre los años 1993 y 2000, se le puede atribuir al PCP-M-L-M una capacidad estratégica y militar elevada, pese a la progresiva disminución de sus acciones. Esto puede ser explicado por tres elementos contextuales claves: la captura de Guzmán, el golpe de Fujimori y las elecciones de 1994. Estos hechos definieron la táctica y estrategia de Sendero en base a 'la acción- reacción', tal y como se actuó, en ocasiones, durante el conflicto armado interno55. Ante estos tres sucesos, la virulencia del PCP-Proseguir fue mayor que en periodos de menor cambio político, por lo que podemos afirmar que 'proseguir' se movió en torno a las circunstancias y no a una estrategia predeterminada. Sostenemos que esta es una de las diferencias existentes con el PCP$S L$, pero que, en esta etapa, aún se manifiesta de forma incipiente. Para que esta situación se mantuviese, suscribimos, en parte, lo expuesto por Rospigliosi:

"Las condiciones que permitieron su crecimiento acelerado (Sendero) desde 1980 ya no existe, que el país y el mundo han cambiado. Pero no hay que olvidar que sigue habiendo un caldo de cultivo para la incorporación de jóvenes frustrados a organizaciones subversivas, y que SL no requirió de grandes masas, ni enormes cantidades de armamento, ni mucho dinero para poner en jaque al país"56.

Nelson Manrique, en esta línea, añadirá que:

"Las causas estructurales que permitieron la generalización de la violencia política en el país, por otra parte, no se ha modificado significativamente. El caldo de cultivo sobre el cual nuevos proyectos violentistas pudieran generalizarse continua allí. [...] El provenir no tiene todos los caminos abiertos. Para despejarlos tendrán que realizare transformaciones muy profundas. Y si estas no son emprendidas a partir de una voluntad política

53 CVR, Anexo estadístico, Comisión por la Verdad y la Reconciliación, Lima, 2003, 21.

54 Ibid.

55 Hertoghe, Alain y Labrousse, Alain, Le Sentier lumineux du Pérou : un nouvel integrisme dans le tiers monde, La découverte, París, 1989.

56 Rospigliosi, Fernando, Caretas, Lima, 9 may.1996. 
de resolver los problemas sociales irresueltos, la perspectiva de volver a recurrir de esa forma de continuación de la política que es la guerra seguirá abierta"57.

La estrategia de 'proseguir', aun en esta época, mantuvo su presencia por la inacción del gobierno en los problemas sociales, la coyuntura fujimorista y por la perduración de las estructuras (aunque menguadas) del PCP-SL. Por ello, esta continuación de los años anteriores duró por poco tiempo.

Por lo tanto, hemos señalado que, aunque existiesen ciertas especificidades, los escisionistas, por lo general, exprimieron la senda del PCP-SL. También apreciamos una cierta continuidad en el ámbito geográfico, siendo las zonas de mayor acción guerrillera las mismas que en los doce años anteriores. Así, los departamentos más hostigados por la violencia seguían siendo Ayacucho, Lima, Junín, Puno, San Martín Huánuco, Huancavelica y Apurímac ${ }^{58}$. Debemos reseñar que, de los departamentos mencionados, San Martín y Lima mantuvieron una actividad bélica considerablemente similar a la de los años anteriores. Esto nos indica que los incipientes núcleos estratégicos se sitúan en la capital y en la mayor región cocalera del país; en añadido, esta información nos anticipa la importancia que empezaba a jugar San Martín (especialmente el Huallaga) en su significación como foco guerrillero.

El resto de las zonas, estratégicamente claves en los años anteriores, sufrirán un progresivo abandono por parte de la nueva dirección senderista, como también por el ya imparable avance de los Comités de Autodefensa Campesina (CAD), rondas y de las fuerzas del Estado. Este abandono del campo en pro de la ciudad entra en contradicción con el propio ideario de Feliciano, que años antes había defendido ante Guzmán una postura agrarista de la estrategia armada59.

Pese a todas estas consideraciones, no se consumó un cambio significativo de estrategia entre el PCP-M-L-M y el PCP-SL, ya que el primero mantuvo el mismo patrón de modalidad de acción subversiva que el segundo. Es decir, aún predominaban las incursiones armadas, el ataque a poblados y el ataque a las FFAA60. Del mismo modo,

57 Manrique, Nelson, El tiempo del miedo, 260-261.

58 Policía Nacional del Perú (PNP), "Perú: estadísticas de violencia y seguridad 1998 - 1999", PNP, 1999. http://proyectos.inei.gob.pe/web/biblioineipub/bancopub/Est/Lib0061/indice.htm(Consultado el 10/01/2018).

59 Caretas, Lima, 9 may, 1996.

60 Policía Nacional del Perú (PNP), "Perú: estadísticas de violencia y seguridad 1998 - 1999", PNP, 1999. http://proyectos.inei.gob.pe/web/biblioineipub/bancopub/Est/Lib0061/indice.htm(Consultado el 10/01/2018). 
las víctimas de estas acciones seguían el mismo patrón que en los años anteriores. Los principales objetivos continuaban siendo los civiles, policías, militares y representantes políticos.

Hechas estas consideraciones, sostenemos que para 'proseguir' sostener toda la capacidad bélica y de organización después de 1992 fue un desafío fracasado, debido a que mantener una correlación de fuerzas similar a la del PCP-SL antes de 1992 era una utopía después del duro golpe de la captura de Guzmán. El aparato militar del PCP-M-L-M y su dirección se vieron sobredimensionados para mantener forzosamente la misma estrategia en una nueva realidad objetiva, lo que supuso, ahora sí, la retirada estratégica de 1996. Sendero Rojo se desalentó poco a poco al no poder mantenerse a sí mismo. Si bien de 1992 a 1995 la facción proseguir mantuvo una continuidad estratégica con el PCP-SL, es a partir de 1996 donde hallamos una mutación ideológica y estratégica hacia el foquismo. Formulamos la especificidad de esta etapa en base a dos factores: el aislamiento y la mutación estratégica.

En 1996 Sendero Rojo estaba sufriendo un fuerte hostigamiento por parte de las fuerzas estatales. En este contexto, dirigentes y cuadros senderistas comenzaban a caer del mismo modo que sucedió en 1992. La fragmentación de fuerzas era un hecho. La ofensiva estratégica empezaba a frenarse y la solución sería el aislamiento a modo de foco. Sucesos como la caída progresiva del Comité Metropolitano de Lima y la desbandada generalizada ${ }^{61}$ incentivaron la retirada estratégica hacia el campo. Un documento de la embajada americana de Lima, filtrado por WikiLeaks, nos revela que el mismo Feliciano había iniciado una huida e, incluso, estaba sitiado. Dice así:

"A peruvian military officer in the Ayacucho region recently told us that his unit believes Oscar Ramirez Durand (a.k.a. "Feliciano"), the most important Sendero Luminoso (Shining Path - SL) leader outside of jail, is still cornered with thirty to fifty cadres in a broken, wooded region near the intersection of the Montaro and Apurimac rivers. this is not the first time that peruvian officials have expressed their belief that the sl leader was trapped -- somehow in the past he has always managed to escape. end summary. 3. (c) colonel Darwin Rengifo Rios, second in command at the ayacucho military base, told us june 4 that the Peruvian military had succeeded in cornering Sendero Luminoso

61 CVR, Informe final, 280. 
(SL) leader "Feliciano" with thirty to fifty Shining Path cadres in the triangular region southwest of the intersection of the Apurimac and Montaro rivers. the roughly 900 square"62.

Esto nos indica hasta qué punto se constreñía el cerco policial que, incluso, llegó a su cabeza más reconocida, Feliciano. Por esto mismo, el aislamiento hacia dos de las regiones más inexpugnables, el Valle del Alto Huallaga (VAH) y el Valle de los ríos Apurímac, Ene y Mantaro (VRAEM), fue una realidad. Feliciano arrastró a los dirigentes hacia posiciones dignas del guevarismo. El aislamiento en el VAH y en el VRAEM entró dentro de los planteamientos foquistas de "conservar una base de operaciones fuerte", "el territorio tiene que ser lo más abrupto posible" y "la estrategia tiene que ser como una colmena: sale de un foco para esparcirse"63.

En cierta manera, el foco significó el retroceso en buena parte de los 'logros' conseguidos por el PCP-SL y por 'proseguir' hasta 1996. Muestra de ello es la disminución de sus actividades armadas. De 1996 a 1998 se produjeron un total de 1.623 acciones subversivas, y entre 1993 y 1994, las acciones sumaron un total de 2.79164. Esto indica la pérdida progresiva de capacidad armada. Esto se suma a que, tal y como comentábamos, se abandonó la ciudad, como confirma el hecho de que el número de acciones subversivas en la sierra supera ya a partir de 1995 al número de acciones en la costa, evidenciando el repliegue defensivo. En concreto, los departamentos donde más creció la cantidad de muertos y desaparecidos, es decir, donde encontramos más presencia, fueron Junín, San Martín, La libertad y Ayacucho, zonas en su mayoría rurales65. Del mismo modo, de 1996 a 1998 se observa cómo las acciones en Lima caen en declive, y departamentos como Huánuco o San Martín tienen preponderancia66. Esto significa, pues, el paso de la ciudad al campo. Así es cómo se relega el papel de las ciudades a un segundo plano, quitándoles el potencial revolucionario, como el mismo Guevara afirmaría "el guerrillero es un revolucionario agrario"67.

62 Wikileaks, "Sendero luminoso leader "feliciano" cornered -- yet again"., Wikileaks, 2012, https://wikileaks.org/plusd/cables/97LIMA4928_a.html (Consultado el 3/05/2018)

63 Guevara, Ernesto, La guerra de guerrillas, Txalaparta, Nafarroa, (1960), 2017, 23-43-76.

64 Policía Nacional del Perú (PNP), “Perú: estadísticas de violencia y seguridad 1998 - 1999", PNP, 1999. http://proyectos.inei.gob.pe/web/biblioineipub/bancopub/Est/Lib0061/indice.htm(Consultado 10/01/2018).

65 CVR, Anexo estadístico, 22.

66 Policía Nacional del Perú (PNP), “Perú: estadísticas de violencia y seguridad 1998 - 1999", PNP, 1999. http://proyectos.inei.gob.pe/web/biblioineipub/bancopub/Est/Lib0061/indice.htm(Consultado el 10/01/2018).

67 Guevara, Ernesto, La guerra de guerrillas, 16. 
De igual forma, la disminución de las acciones guerrilleras y del número de víctimas y desparecidos totales a manos de los senderistas rojos se explica por el declive progresivo que sufrió la organización, por su falta de dirección y por el cerco policial al que estaba sometida. Ahora bien, hay autores que defienden la tesis de que la disminución de la violencia y la estrategia del PCP-M-L-M está basada en una modificación de su trato con la población, produciéndose un nuevo enfoque social basado en una relación más cercana y no coercitiva con las masas ${ }^{68}$. La tesis sostenida por estos autores, desde mi pensar, peca de generalista y esencialista, anteponiendo lo particular a lo general, de lo subjetivo a lo objetivo. Des de luego, no negamos la existencia de una 'reorientación' estratégica parcial en el trato con la población. Empero, esta 'nueva' estrategia entraría dentro de una nueva línea de masas caracterizada por ser más laxa con la población, siendo más acorde con las experiencias foquistas.

La información proveniente de la prensa, de forma sucinta, nos da habida cuenta de esta nueva estrategia del PCP-M-L-M. En la agencia Inter Press Service se afirmó por partida doble que "fuera de la selva sendero está haciendo trabajo proselitista, con un mayor énfasis en barriadas urbanas que en el campo porque las rondas campesinas [...] no permiten a los senderistas retornar fácilmente"69, junto con "Sendero Rojo trata de recuperar sus bases de apoyo en Ayacucho"70.

De la misma forma, hallamos en un reportaje fechado en 1994, en el medio El País, que "Las menguadas tropas de Feliciano atemperan su cruenta predica y reconocen errores", y actúan con el lema "Discute, discute, argumenta, no te calles"71. En la revista Caretas, siguiendo con lo expuesto por El País, afirman que "A pesar de que los seguidores de Oscar 'Feliciano' Ramírez Durand prediquen una nueva estrategia sin los 'errores' de antes, gran parte de la población no les cree"72. Y "En estos días se puede observar cómo los hombres de 'Feliciano' en la selva central hacen discursos ante los pobladores, explicándoles lo que describen como los errores

68 Esta visión la encontramos en los siguientes trabajos: Burt, Jo-Marie y López Ricci, José, "Perú: Shining Path after Guzmán", 6-9; Ríos, Jerónimo y Sánchez, Marté, Breve historia de Sendero Luminoso, Catarata, Barcelona, 2018; Rojas, Isaías, "Y a todo esto ¿en qué está Sendero Luminoso?", Revista Idéele, no112, 1998, 17-21; Bourllaud, Jean, Dolfus, Olivier y Gondard, Pierre, "Pérou: Le Haut Huallaga de la coca à l'abandon", Problemes d'Amerique Latine, no28, 1998, 109-123.

69 Lama, Abraham, Inter Press Service, Lima, 12 mar. 1997.

70 Lama, Abraham, Inter Press Service, Lima, 17 jun. 1999.

71 Aznárez, Carlos, El País, Madrid, 9 jun. 1999.

72 Caretas, Lima, 23 oct, 1997. 
cometidos en el pasado, en alusión a los asesinatos de dirigentes populares"73. Como último ejemplo, el periodista León-Jimmy Torres nos informa de que, en la captura de dos senderistas, se encontraron directrices en favor de intensificar el trabajo político y reforzar el movimiento clasista barrial74.

A partir de estos ejemplos, $\dot{2}$ se puede generalizar un cambio total de la actitud del PCP-Proseguir respecto de la población? Nosotros respondemos que, de forma parcial, se puede afirmar la existencia de un cambio de línea de masas. Sobre todo, en las zonas donde mayor control ejerce Sendero Rojo. Empero, hay que rehusar una generalización de esta nueva estrategia, ya que las acciones de estos tienen un cariz ambivalente y cambiante, al no existir una centralización efectiva después de 1996, ya que difícilmente se dirigieron de forma eficaz todas las acciones fuera del VAH Y del VRAEM. Así, la nueva línea de masas no es uniforme para todo el territorio, sino que específicamente lo sería para aquellas zonas con más presencia, como son Ayacucho, Apurímac, Huánuco y San Martín.

En contraposición al argumento de la unificación de la línea de masas de Sendero Rojo, encontramos acciones senderistas que contradicen esa tesis y que mantienen una belicosidad y distan de la línea de masas nombrada con anterioridad. Mientras que el PCP-M-L-M en el Huallaga y en la selva central se manifestaba en contra de los asesinatos selectivos contra la población, en Lima, concretamente en las barriadas, ejercía una exacerbada violencia contra líderes populares, obreros y población civil.

Los ataques hacia líderes populares disminuyeron sus víctimas, pero mantuvieron una dinámica de temor. Así fue como asesinaron a Pascuala Rosado en 1996, asesinaron a 9 tenientes gobernadores, a 1 concejal, 2 alcaldes distritales, 1 alcalde provincial, 10 bomberos, 176 civiles y 2 jueces 75 . Además, añadimos actividades poco propias del PCP-SL, que fueron utilizadas por Sendero Rojo, como sucedió con los secuestros. Los secuestros, y la tentativa de ellos, son una actividad propia del terrorismo urbano y de las organizaciones criminales, pero fueron implementados por el PCP-M-L-M. Hallamos casos de secuestros de jóvenes en el Huallaga, planes para secuestrar a hijos de almirantes y secuestros de miembros de la

73 Caretas, Lima, 15 ago. 1996

74 Torres, León-Jimmy, Caretas, Lima, 8 ago. 1996.

75 Policía Nacional del Perú (PNP), “Perú: estadísticas de violencia y seguridad 1998 - 1999 ”, PNP, 1999. http://proyectos.inei.gob.pe/web/biblioineipub/bancopub/Est/Lib0061/indice.htm(Consultado el 10/01/2018). 
comunidad Ashánika76. De hecho, existe una correlación de esta actividad del PCPProseguir con el incremento de la modalidad del secuestro con fines criminales en el Perú77. También debemos añadir la realización de acciones indiscriminadas 78 , como lo fueron los atracos a sucursales bancarias79, asalto y saqueo de camiones y fábricas ${ }^{80}$, y el ametrallamiento contra un avión proveniente de Estados Unidos ${ }^{81}$, entre otras acciones.

Según la cosmovisión comunista, para mantener vínculos con las masas, hay que actuar de acuerdo con sus necesidades y deseos, y hay que actuar con paciencia hasta que hayan adquirido conciencia de la necesidad de un cambio y tengan el deseo de llevarlo a cabo82. Así, las masas son los verdaderos héroes83. También, la conducta con la población debe estar reglada por un gran respeto a todas las tradiciones y normas de la gente. No debe ajusticiarse sin dar oportunidad de descargo al reo, salvo momentos especiales 84 . Las acciones citadas con anterioridad distan mucho de los verdaderos planteamientos para atraer a la población, por lo que debemos matizar que el PCP-M-L-M haya experimentado una 'reorientación social'. Más bien ha modificado, en las zonas donde tiene mayor influencia, su violencia hacia la población civil con una incipiente 'línea de masas', más constructiva y menos coercitiva.

Por último, dejando de lado la estrategia empleada con la población, debemos señalar los cambios en las acciones militares, aspecto relacionado directamente con el foquismo. Como anteriormente se ha señalado, a partir de 1996 el PCP-Proseguir entró en una estrategia foquista, con dos núcleos relevantes: el VAH y el VRAEM. Las zonas restantes, especialmente las urbanas, fueron abandonadas. Así, se observa cómo los paros armados disminuyeron considerablemente85. Por el

76 Ibarz, Joaquim, La Vanguardia, Barcelona, 23 jul. 1995, p. 10; Flores, Félix, La Vanguardia, Barcelona, 20 may. 1997, p. 2-3; Agencia Efe, La Vanguardia, Lima, 23 may. 1993, p. 8.

77 Caretas, Lima, 22 feb.1996.

78 Este 'nuevo' accionar de Sendero Rojo fue incluso criticado por miembros del PCP-SL, los cuales calificaron de 'bandolerista' las actividades del bloque 'escisionista'. Véase: Rénique, José Luis, Las 'luminosas trincheras de combate' de Sendero Luminoso del Perú, 116.

79 Agencia Efe, $A B C$, Lima, 17 dic. 1993, p. 37.

80 Jiménez, Benedicto, Inicio, desarrollo y ocaso del terrorismo en el Perú, 2: 25-30.

81 Reuters, La Vanguardia, Barcelona, 23 ene. 1993, p.5.

82 Zedong, Mao, El libro rojo, 103.

83 Zedong, Mao, El libro rojo, 99.

84 Guevara, Ernesto, La guerra de guerrillas, 31.

85 Policía Nacional del Perú (PNP), "Perú: estadísticas de violencia y seguridad 1998 - 1999”, PNP, 1999 http://proyectos.inei.gob.pe/web/biblioineipub/bancopub/Est/Lib0061/indice.htm(Consultado el 10/01/2018). 
contrario, establecerse en las zonas más recónditas cumplía la máxima del Che Guevara de "Todos los medios favorables, todas las facilidades para la vida del hombre hacen tender a éste a la sedentarización: en la guerrilla sucede lo contrario; mientras más facilidades haya para la vida del hombre, más nómada, más incierta será la vida del guerrillero"86.

Así es como el Huallaga y el VRAE devienen centros guerrilleros de los cuales salen las acciones armadas. Respecto a los años anteriores, la estrategia, afirmamos, ha evolucionado hacia un abandono de los ataques contra las infraestructuras económicas, cooperativas, medios de comunicación y centros mineros. En consecuencia, se potencia una actuación directa contra las fuerzas del orden, minimizando el contacto con los trabajadores. Por ello, este cambio, y aquí radica el punto principal del foquismo, se dedica casi exclusivamente a luchar contra el Estado a modo de invasión armada, control de poblados, enfrentamientos armados, hostigamientos y ataques directos y, por último, emboscadas.

El desplazamiento de Sendero Rojo a las zonas cocaleras del VAH y del VRAEM también supuso una mayor interacción con la producción de coca y el narcotráfico. Parte de la historiografía existente durante el conflicto armado interno estableció que tanto el PCP-SL como Sendero Rojo tuvieron una implicación directa y total con el narco, por lo que fueron calificados como 'narcoterroristas' 87 . Estas posturas iban en la línea de que Sendero Rojo participó directamente en todas las fases de la coca, por lo que establecerían alianzas y consentirían toda la práctica ilícita y criminal.

Sin embargo, a partir de las conclusiones de la CVR y de nuevos estudios se afirmó que Sendero Rojo inició un acercamiento no buscado, sin finalidad en la distribución ni el contrabando; únicamente estaba presente en la percepción del impuesto revolucionario88. También participaría en el control de precios y en la tasación de productos. En este sentido, tales afirmaciones desestimarían una implicación directa, pero sí establecerían un contacto con los narcotraficantes

86 Guevara, Ernesto, La guerra de guerrillas, 43.

87 Algunos ejemplos sobre tal planteamiento son: Chamba, $\mathrm{F}$ et al., "Coca et violence: le témoignage du Alto Huallaga au Pérou", Aurepart, n.26, 2003, 157-171; Hertoghe, Alain y Labrousse, Alain, Le Sentier lumineux du Pérou: un nouvel integrisme dans le tiers monde.La découverte, París, 1989; Moreno, José Manuel. "Sendero Luminoso, narcoterrorismo y seguridad en el Perú", GESI, n3, 2016, http://www.seguridadinternacional.es/?q=es/content/ sendero-luminoso narcoterrorismo-y-seguridad-en-el-perú (Consultado el 17/12/2017); Strong, Simon, Shining Path: the world's deadliest revolutionary force, Harper Collins Publishers, London, 1992; Tarazona Sevillano, Gabriela, Sendero Luminoso and the Threat of Narcoterrorism, The Washington Papers, Washington, 1990.

88 CVR. Informe Final, 759. 
basado en una relación de poder similar89. Esta postura fue incluso ratificada por el poder judicial del Perú en 2018, el cual, en contra incluso de la Fiscalía, sentenció que las acusaciones de narcotráfico carecían de fundamento y los testimonios eran indirectos y no corroborados 90 .

Finalmente, afirmamos que en esta etapa el PCP-M-L-M se adaptó a las circunstancias de su retirada estratégica al campo. La retirada y el establecimiento en el agro cambiaron su estilo maoísta de bases de apoyo, al evitar el contacto directo con la población no estableciéndose en centros poblacionales, negándose a armar a los campesinos, actuando casi exclusivamente en un mismo territorio y, sobre todo, no planteando una estrategia a largo plazo y sometiendo los avances a la voluntad del líder.

\section{Conclusiones}

Una vez realizado el estudio sobre Sendero Rojo, establecemos las conclusiones pertinentes y los aportes de la investigación. Dicha investigación nos ha llevado a establecer que, primero como línea y segundo como partido, el PCP-M-L-M sufrió un proceso de mutación ideológica, estratégica y organizativa. Este proceso tiene dos ejes claves que han guiado la investigación.

El primero hace mención a que Sendero Rojo tiene que ser estudiado como un objeto de estudio propio y autónomo, debido a que ya en 1992 empezó a convertirse y erigirse como independiente del PCP-SL; y así fue cómo, a partir de 1995, la separación se consumó de manera significativa. Con la regionalización y el desarrollo del foquismo, reflejado en las nuevas práctica militares, sociales y políticas, este proceso se hizo más que evidente. Así, asumimos que el estudio de Sendero Rojo debe

89 Esta tesis es sostenida por: Bourllaud, Jean, Dolfus, Olivier y Gondard, Pierre, "Pérou: Le Haut Huallaga de la coca à I'abandon", Problemes d'Amerique Latine, no28, 1998, 109-123. Navarrete-frías, Carolina y Thoumi, Francisco. E., Drogas Ilegales y Derechos Humanos de Campesinos y Comunidades Indígenas: el caso de Perú, ONU, París, 2005; Novak, F et al., Niños, Niñas y Adolescentes en las Zonas Cocaleras del VRAE y el Alto Huallaga, Pontificia Universidad Católica del Perú, Lima, 2011; Ríos, Jerónimo y Sánchez, Marté, Breve historia de Sendero Luminoso, Catarata, Barcelona, 2018; Obando, Enrique, "El tráfico ilícito de drogas en el Perú, cuarenta años después", Revista temática, Centro de altos estudios nacionales, n2, 17-45, 2016.

90 Quispe, Diego, "Poder Judicial exculpó a Sendero Luminoso de delitos de narcotráfico", La República, 2018, https://larepublica.pe/politica/1319620-judicial-exculpo-sendero-luminoso-delitos-narcotrafico/ (Consultado el 26/09/2018). 
responder a su propia esencia, y no en oposición al PCP-SL.

El segundo eje reside en que el proceso de escisión, creación, desarrollo y consolidación de Sendero Rojo responde a la dialéctica interna del PCP-SL. Es decir, detrás de esta nueva organización, se esconde toda la tradición de lucha interna desarrollada en el seno del PCP-SL. Esta, después de la captura de Guzmán, es acelerada y estalla por la delicada coyuntura política inmediata. Así pues, entendemos la mutación ideológica, organizativa y estratégica como un proceso dialéctico, largoplacista, progresivo, derivado de la inexistencia de una continuidad con Abimael Guzmán, de proseguir con la lucha armada, de la nueva dirección de Feliciano y del progresivo debilitamiento militar, especialmente a partir de 1995.

Hechas estas consideraciones, debemos señalar los aportes que hemos llevado a cabo con la presente investigación. Estos aportes atañen de forma temática a los tres apartados del trabajo: ideología, organización y estrategia.

El primer aporte hace mención a que hemos constatado, mediante el estudio de los textos de Sendero Rojo, que se produce una diferenciación ex profeso del PCPSL. Este proceso fue paulatino de 1992 a 1999, con el punto de inflexión localizado en 1995, momento en que las diferencias entre ambas formaciones se volvieron irreconciliables, ya sea por la regionalización o por el avance de las negociaciones de paz entre Guzmán y Fujimori. Empero, los debates no surgieron en 1992, sino que las discusiones se venían desarrollando desde años antes, hecho que explica la rápida escisión y fraccionamiento. Del mismo modo, el énfasis de Sendero Rojo en publicar sus comunicados y en hacerse notar mediante atentados, hace remarcar su voluntad de diferenciación y, en definitiva, de consolidarse y autoafirmarse como un sujeto autónomo (heredero del PCP-SL) que desea proseguir la lucha armada y, al mismo tiempo, hace autocrítica del pasado.

El segundo aporte reside en la organización. Se ha constatado que Sendero Rojo presentó, en similitud con lo referente a la ideología, un estructura similar a la del PCP-SL hasta 1995, año en que se produjo la regionalización y el abandono de los núcleos urbanos en favor del campo. De este modo, se mantuvieron las mismas dinámicas verticales y de jerarquización estereotipada del PCP-SL. En similitud, se ha demostrado en los primero años después de la captura de Guzmán, la organización y capacidad fue similar a la llevada a cabo con anterioridad a 1992 e, incluso, se observa una mayor belicosidad y virulencia en las acciones militares. Por el contrario, 
en el ámbito de producción teórica, es decir, en la organización de la vanguardia, la gestión fue deficiente. A medida que la regionalización fue consolidándose, la organización, en consecuencia, se vio modificada por la dirección unívoca de un jefe militar. En otras palabras, las estructuras organizativas de Sendero (heredadas por Sendero Rojo) no pudieron ser mantenidas en el tiempo, los maoístas se vieron sobrepasados.

El tercer y último aporte de este artículo nos indica que el PCP-M-L-M mantuvo una posición menos directa y, en consecuencia, más laxa con la población, ya que regionalizó su campo de actuación. Este actuar se generalizó por la menor involucración política de las comunidades campesinas, puesto que basó sus actividades en la defensa del precio de la coca y en la confrontación (casi única) contra las fuerzas de seguridad, evitando el contacto con trabajadores y campesinos. En oposición, la actuación en los núcleos urbanos hasta 1995, como en los casos anteriores, fue similar a la del PCP-SL. Incluso podemos señalar una estrategia poco usual, como fueron los secuestros, atracos a entidades bancarias, asalto de camiones etc., modalidades de accionar que tienen más en común con el bandolerismo social que con la praxis marxista-leninista-maoísta.

En definitiva, constatamos que el PCP-M-L-M o Sendero Rojo, para el periodo de 1992 a 1999, pero especialmente entre 1995 a 1999, entró en un proceso de abandono de la GPP, con el desarrollo de posturas propias del foquismo; produciéndose así una mutación en lo referente a la ideología, organización y estrategia. Esto provocó que Sendero Rojo tuviese un desarrollo autónomo e independiente del PCP-SL con cierta rapidez.

Fecha de recepción: 30/09/19

Aceptado para publicación: 10/02/20 


\section{Fuentes}

Antezana, Jaime, “El senderismo real está en el Movadef”, Perú21, 2009, https://peru21.pe/politica/jaime-antezana-senderismo-real-movadef-193743 (Consultado el 3/01/2018).

Quispe, Diego, "Poder Judicial exculpó a Sendero Luminoso de delitos de narcotráfico", La República, 2018, https://larepublica.pe/politica/1319620-judicial-exculpo-senderoluminoso-delitos-narcotrafico/ (Consultado el 26/09/2018).

Policía Nacional del Perú (PNP), "Perú: estadísticas de violencia y seguridad 1998 1999", PNP, 1999, http://proyectos.inei.gob.pe/web/biblioineipub/bancopub/Est/Lib0061/indice.htm (Consultado el 10/01/2018).

PCP-M-L-M, "iQue la mitad que sostiene el cielo sea ejemplo una vez más, Hoy en la nueva etapa del partido!” $P C P-M-L-M, 1994$, http://www.cedema.org/ver.php?id=3527 (Consultado el 29/03/2018).

PCP-M-L-M, “'Superar el recodo, desarrollando la guerra popular!”, PCP-M-L-M, 1995, http://www.cedema.org/ver.php?id=3527 (Consultado el 29/03/2018).

PCP-M-L-M, "¡Contra la dictadura genocida y vendepatria, persistir en la guerra popular!", PCP-M-L-M, 1995, http://www.cedema.org/ver.php?id=718 (Consultado el 30/03/2018).

PCP-M-L-M, "Informe político", $P C P-M-L-M, 1996$, http://www.cedema.org/ver.php?id=723 (Consultado el 29/03/2018).

WikiLeaks, "Sendero luminoso leader "feliciano" cornered -- yet again"., Wikileaks, 2012, https://wikileaks.org/plusd/cables/97LIMA4928_a.html (Consultado el 3/05/2018)

\section{Referencias bibliográficas}

Burt, Jo-Marie y López Ricci, José, "Peru: Shining Path after Guzmán”, NACLA Report on the Americas, v.3, n.28, 1994, 6-9.

Bourllaud, Jean, Dolfus, Olivier y Gondard, Pierre, "Pérou: Le Haut Huallaga de la coca à l'abandon", Problemes d'Amerique Latine, no28, 1998, 109-123.

CVR, Informe Final, Comisión de la Verdad y Reconciliación, Lima, 2003.

CVR, Anexo estadístico, Comisión de la Verdad y Reconciliación, Lima, 2003.

Chamba, F et al., "Coca et violence: le témoignage du Alto Huallaga au Pérou", Aurepart, n.26, 2003, 157-171.

Clauswitz, Carl Von, De la guerra, Labor, Barcelona, (1832),1992.

Guevara, Ernesto, La guerra de guerrillas, Txalaparta, Nafarroa, (1960), 2017. 
Hertoghe, Alain y Labrousse, Alain, Le Sentier lumineux du Pérou : un nouvel integrisme dans le tiers monde, La découverte, París, 1989.

Jiménez, Benedicto, Inicio, desarrollo y ocaso del terrorismo en el Perú, 2 vols., Sanki, Lima, 2000.

Lenin, Vladimir Ilich, Materialismo y empiriocriticismo, Grijalbo, Barcelona, (1908), 1975.

Lenin, Vladimir Ilich, ¿Qué hacer?, Debarris, Barcelona, (1902), 2013.

Manrique, Nelson, El tiempo del miedo: La violencia política en el Perú 1980-1996, Fondo Editorial del Congreso del Perú, Lima, 2002.

Moreno, José Manuel, "Sendero Luminoso, narcoterrorismo y seguridad en el Perú", GESI, n3, 2016, http://www.seguridadinternacional.es/?q=es/content/sendero-luminoso narcoterrorismo-y-seguridad-en-el-perú (Consultado el 17/12/2017).

Navarrete-frías, Carolina y Thoumi, Francisco, Drogas Ilegales y Derechos Humanos de Campesinos y Comunidades Indígenas: el caso de Perú, ONU, París, 2005.

Novak, F et al., Niños, Niñas y Adolescentes en las Zonas Cocaleras del VRAE y el Alto Huallaga, Pontificia Universidad Católica del Perú, Lima, 2011.

Rénique, José Luis, La voluntad encarcelada. Las 'luminosas trincheras de combate' de Sendero Luminoso del Perú, Instituto de Estudios Peruanos, Lima, 2003.

Ríos, Jerónimo y Sánchez, Marté, Breve historia de Sendero Luminoso, Catarata, Barcelona, 2018.

Rojas, Isaías, "Y a todo esto ¿en qué está Sendero Luminoso?”, Revista Idéele, no112, 1998, 17-21.

Strong, Simon, Shining Path: the world's deadliest revolutionary force, Harper Collins Publishers, London, 1992.

Tarazona Sevillano, Gabriela, Sendero Luminoso and the Threat of Narcoterrorism, The Washington Papers, Washington, 1990.

Tsé-Tung, Mao, La guerra revolucionaria, Grijalbo, México, (1936), 1971.

Zedong, Mao, El libro rojo, Espuela de plata, Sevilla, (1964), 2014. 